\title{
Advancing Social Simulation: Lessons from Demography
}

\author{
Eric Silverman ${ }^{1}$, Jakub Bijak ${ }^{1}$, Daniel Courgeau ${ }^{2}$ and Robert Franck ${ }^{3}$ \\ ${ }^{1}$ University of Southampton, Southampton, UK \\ ${ }^{2}$ Institut national d'études démographiques, Paris, France \\ ${ }^{3}$ Université catholique de Louvain, Louvain-la-Neuve, Belgium \\ e.silverman@soton.ac.uk
}

\begin{abstract}
Previous work has proposed that computational modelling of social systems is composed of two primary streams of research: systems sociology, which is focused on the generation of social theory; and social simulation, which focuses on the study of real-world social systems. Here we argue that the social simulation stream stands to benefit from recent methodological and theoretical advances in demography. Demography has long been an empirically focused discipline focused primarily on mathematical modelling; however, agentbased simulation have proven influential of late as demographers seek to link individual-level behaviours to macro-level patterns. Here we characterise this shift as a move toward system-based modelling, a paradigm in which the scientific object of interest is neither the individual nor the population, but rather the interactions between them. We first describe the four successive paradigms of demography: the period, cohort, event-history and multilevel perspectives. Then we examine how system-based modelling can assist demographers with several major challenges: overcoming complexity in social research; reducing uncertainty; and enhancing theoretical foundations. We propose that this new paradigm can enhance the broader study of populations via social simulation.
\end{abstract}

\section{Introduction}

Understanding the complex and multi-layered processes at play in social systems is far from a simple task, and in recent years research efforts have focused on studying these systems using computer simulation. Taking inspiration from Alife and related areas of complex systems science, the field of social simulation has taken shape as a discipline devoted to the study of social systems as complex, non-linear systems composed of interacting agents.

Social simulation has grown steadily over the last decade as social scientists grow increasingly attracted to the idea of representing the complexities of the social realm using simulation (Billari and Prskawetz, 2003). However, questions remain regarding how to best utilise these tools in different contexts. Social simulation offers tremendous flexibility and provides scope for experimentation beyond simple prediction (Epstein, 2008), but what methodological frameworks can guide us in our investigations? Can complex systems simulation approaches ever be closely linked with traditional empirically-focused social science methods?

\section{Systems Sociology vs. Social Simulation}

Previous work has examined the core questions of social simulation in-depth using several case studies as exemplars of different simulation research programmes (Silverman and Bryden, 2007). This work proposed that what we refer to as social simulation can be more accurately represented as two separate streams of research: systems sociology and social simulation.

Systems sociology encompasses the drive to develop stronger social theories regarding the development and evolution of societies. The use of simulation allows system sociologists to investigate abstract social systems in an effort to understand the core communicative processes that lead to higher-level social complexity; this instantiates the kind of investigations spearheaded by sociological theorists like Niklas Luhmann (1984).

In contrast, social simulation uses computational methods to examine specific elements of social systems, frequently based on real-world examples such as residential segregation (Schelling, 1978), or social care in ageing populations (Silverman et al., 2013). This approach benefits from a closer connection to social phenomena for which usable data is actually available, which can allow for easier validation of simulation results. However, parameterising simulations using the available data is still difficult and is not a straightforward process.

We propose that developing a consistent methodological framework for social simulation is a difficult but necessary task. Simulation approaches allow the modeller to investigate the link between micro-level behaviour and macro-level patterns, which is of great interest to social scientists. In order to further our methodological ambitions, we suggest that social simulation researchers take on board insights from the field of demography, where recent developments have indicated a shift toward a systems-level take on social phenomena that can inform and enhance social simulation efforts. 


\section{Examining Demography}

Demography as a research field has existed for over 350 years, and during that time has advanced through several successive paradigmatic changes. From the early 'political arithmetics' of Graunt (1662) to current multilevel methods (Courgeau and Franck, 2007), demography has developed increasingly sophisticated means for the examination of population data. The recent popularity of agent-based approaches has prompted a shift in focus toward individual behaviours and interactions and away from strictly populationlevel insights (Burch, 2003b; Silverman et al., 2011). In this section we will outline the developments in demography over the last three and a half centuries and provide a summary of the primary paradigm shifts that have taken place.

In this discussion we will use a different definition of the word 'paradigm' than those developed by Kuhn (1962). Instead we take inspiration from Granger (1994) and define paradigms as a means to describe the relationship between an observed phenomena and the scientific object. In the case of demography, the scientific object is primarily populationlevel change - but as we shall see, recent developments indicate a shift in this fundamental perspective.

Courgeau and Franck (2007) identify four such paradigms in demography: cross-sectional (period), longitudinal (cohort), event history and multilevel. Each of these paradigms expands upon and addresses weaknesses of previous paradigms, but we must emphasise that the demography displays cumulativity in that new paradigms enhance new avenues of enquiry but do not replace or eliminate previous paradigms. This is similar to the cumulativity we see in other disciplines, such as physics, in which Einstein's relativity addresses weaknesses in Newton's classical mechanics, but nevertheless classical mechanics remains a more than sufficient tool for a great variety of problems in physics.

We note that this perspective on cumulativity can be seen as a departure from Kuhn, who argued that we should "take it for granted that the differences between successive paradigms are both necessary and irreconcilable" (1970). However, following on from Courgeau (2010), we posit that the knowledge generated by successive paradigms displays a non-linear cumulativity. Agazzi has argued that scientific theories focus on a small set of objects, some of which are referential and can differ between theories in the same discipline, while others are contextual and relate to the core concepts which can remain invariant between theories and therefore allow for theory comparison. Agazzi then moves beyond Kuhn and suggests that "scientific progress does not consist in a purely logical relationship between theories, and moreover it is not linear.... and may even be interpreted as an accumulation of truth" (1985).

Within demography, we will see through our historical analysis that the four successive paradigms each surpass certain shortcomings of the previous, and yet these previous paradigms are still in active use. This is because demogra- phers using these different methodologies continue to operate in the same context, even while the perceived relationships between demographic events of interest change significantly from one paradigm to another. This suggests that demographic paradigms do not eliminate previous approaches but instead display a non-linear cumulativity of knowledge as new theories sit alongside one another in the broader context (Courgeau, 2010).

Next we will outline the history and structure of demography, and then we will make our case for a fifth paradigm: system-based modelling, which is focused on interactions within population systems.

\section{Paradigms in Demography}

Demography has its origins in the work of Graunt (1662), who is considered to be the first to apply the scientific method to the study of human populations. Graunt was able to abstract away the concepts of mortality, fertility and morbidity from individuals, and study these as objects of scientific scrutiny in and of themselves. He also connected studies of probability with these examinations of populations, leading to the first studies of events happening to statistical individuals as defined by Courgeau (2012). We characterise these early investigations, and indeed most of the following 200 years of demography, as part of the cross-sectional paradigm, in which population-level events are observed and measured according to historical time. As suggested by Courgeau (2007), we see the cross-sectional paradigm defines the social facts of a given period as existing independently of the individuals affected.

The next paradigm, longitudinal analysis, came about through American researchers including Ryder (1951) and was eventually formalised by Henry (1959). Under this paradigm the demographer studies the occurrence of a single event during the life of a cohort (a group of individuals experiencing a particular event during a particular stretch of time). However, this approach requires that this cohort be homogenous and the phenomenon under study must be independent (Courgeau, 2007). The restrictive nature of this approach meant that a new paradigm was soon required in order to allow the study of heterogeneous cohorts and to allow for dependencies between phenomena of interest.

That paradigm soon manifested in the form of the eventhistory analysis approach, pioneered by Aalen (1975) following the introduction of general theories of stochastic processes. In this paradigm individuals are seen to follow complex trajectories that depend upon previous events and information they have acquired in the past (Courgeau, 2007). This approach necessitates the use of detailed surveys which can collect data on events and characteristics on an individual level. However, this approach requires significant assumptions when applied to the study of population change; chief among these is the assumption that all individuals are assumed to follow the same random process, the parameters 


\begin{tabular}{|c|c|c|l|}
\hline No. & Paradigm & Period & Key Focus \\
\hline \hline 1 & Period (cross-sectional) & $1662-$ & $\begin{array}{l}\text { Population-level phenomena, observed and measured according } \\
\text { to historical time }\end{array}$ \\
\hline 2 & Cohort (longitudinal) & 1950 s- & $\begin{array}{l}\text { Population-level phenomena, observed and measured along the } \\
\text { lifetime of individual cohorts }\end{array}$ \\
\hline 3 & Event History & $1980 s-$ & $\begin{array}{l}\text { Individual-level phenomena, observed and measured according } \\
\text { to individual time }\end{array}$ \\
\hline 4 & Multilevel & $1980 s-$ & $\begin{array}{l}\text { Individual, population, and interim-level phenomena, observed } \\
\text { and measured from multiple perspectives }\end{array}$ \\
\hline 5 & System-based & $2000 s-$ & $\begin{array}{l}\text { Interactions between population systems of individuals, groups } \\
\text { and institutions }\end{array}$ \\
\hline
\end{tabular}

Table 1: A summary of the four previous paradigms of demography

of which are estimated from the statistical sample of individuals.

In order to introduce different types of groupings of individuals and allow for the influence of a broader social context, demographers turned to the fourth paradigm: multilevel analysis. This context could include socio-economic groupings, social networks, etc. The multilevel approach addresses weaknesses of the event-history approach by allowing for individual behaviour to be constrained by external factors (Courgeau, 2007). While these advantages are significant, multilevel analyses still do not allow for feedback effects (i.e., influences on higher-level behaviour from individual actions), and we shall see how our proposed fifth paradigm addresses these shortcomings.

While we can see a clear progression in demography toward the examination of increasingly complex relationships between social facts at the individual and population levels, each of these paradigms remains in use in certain contexts. Indeed, cross-sectional analyses remain relevant for certain investigations in demography, and this is likely to remain the case for the foreseeable future. In order to examine the complex interactions and feedbacks between the individual level and the population level, however, we need another advancement in methodology. In the next section we outline some of the most pressing challenges facing demography, which will lead to our proposal for the fifth demographic paradigm.

\section{Epistemological Challenges in Demography}

The development of demography in recent decades has been closely tied to debates around epistemological challenges. The advent of the multilevel approach, for example, was a product of extensive discussion amongst demographers regarding the examination of multiple levels of analysis in population science. Currently demographers are taking on the issues of uncertainty and complexity.

Demographic changes are inherently uncertain, as with any other aspect of social systems, although demography appears less susceptible to uncertainty in some respects than some related disciplines (e.g., economics). Demographers contend that this is due to the strong empirical slant of the field, and to the power of the statistical relationships which provide the foundations for much of the research in this area (Xie, 2000). Of course some fundamental aspects of population change are considered more uncertain than others; migration, for example, is thought to be much more uncertain than mortality, given that the former can be strongly affected by a number of factors which can change rapidly, such as economic conditions or legislative changes (Xie, 2000). The open and frequent discussion of uncertainty in demography has led to the 'return of the variance' to demography, which has been of great importance to work within all four paradigms described in the previous section (Courgeau, 2012).

The complexity of social phenomena also increases the uncertainty inherent in demographic change, and so additional methodological innovation is required to address these challenges. While significant debate on complexity in demography has been ongoing (Silverman et al., 2011), there is relatively limited evidence available on the performance of demographic models at varying levels of complexity. When looking at predictive applications, opting for simple models that describe uncertainty in detail may be most appropriate (Bijak, 2010). Prediction is far from the only goal in demography, however - as with social simulation (Epstein, 2008) - so other approaches are still required.

These realisations have led to a move toward instantiating demography as a 'model-based science', as we have seen in population biology (Godfrey-Smith, 2006). Some comparisons between modelling in population biology and in artificial life have outlined how these biological applications have important lessons for modellers in other fields (Bullock and Silverman, 2008). However, models of social reality add additional layers of complexity for the modeller; in particular, formalising the relationships between different levels of social phenomena is far from straightforward (Kluver et al., 2003). 


\section{Addressing Uncertainty}

As these investigations of demographic methodology have progressed, demographers have acknowledged that model uncertainty must be addressed, given the emphasis on formal statistical models in demographic applications. If models themselves are to be acknowledged as sources of additional uncertainty, then the most relevant approach to describing these uncertainties is via Bayesian statistical inference and epistemic probability (see Courgeau, 2012; Raftery, 1995, for discussion). Within this category there are varied approaches to describing model error, from selecting models out of several competing possibilities and related model averaging (Raftery, 1995), to including additional terms for model discrepancy within the modelling process itself (Kennedy and O'Hagan, 2001; O'Hagan, 2006). In addition, Bayesian statistics can allow the modeller to include subjective opinion during the statistical inference process (see, e.g. Bijak, 2010).

Further, Bayesian statistics provides a way to reconcile model-based and empirical approaches by returning to empiricism at a different level of analysis. Computational models, regardless of their structure or complexity, have input parameters and outputs of interest. Various statistical techniques allow for statistical analysis of this mapping, including Bayesian melding (Poole and Raftery, 2000), and Gaussian process emulators (Kennedy and O'Hagan, 2001); for examples of the latter approach in demographic applications, see Bijak et al. (2013) and Silverman et al. (2013). These methods help to alleviate one of the major shortcomings of complex computational models - their relative opacity compared to formal mathematical models - by allowing for an in-depth analysis of the input-output mapping using a formal statistical framework.

Finally, these uncertainty evaluations can be validated by examining various error measures and at empirical frequencies related to predictive intervals with different nominal probabilities. These techniques have been applied in several demographic studies to date, including Bijak (2010), Clark et al. (2012), and Raftery et al. (2012). Quality of calibration can be assessed using scoring rules, as in Gneiting and Raftery (2007).

\section{New Modelling Approaches for Demography}

This growing interest in new modelling methodologies for demography has led to a number of proposals in the literature, many of which have been inspired by agent-based computational approaches (see Billari and Prskawetz, 2003; Bijak et al., 2013; Silverman et al., 2013). Demographers have expressed enthusiasm about the possible ramifications for improving the theoretical foundations of demography (Chattoe, 2003; Burch, 2003a), and some recent work has focused explicitly on developing comprehensive social theories based on demographic foundations (Lutz, 2013). Others have pointed out the benefits of using agent-based models to avoid the over-dependence on increasingly detailed and expensive survey data at the expense of the realism of the explanations offered (Silverman et al., 2011).

The perceived utility of agent-based approaches for explanatory aims has been a significant attraction for demographers (Burch, 2003b; Silverman et al., 2011). Agent-based models are designed to represent the impact of individual behaviours on macro-level patterns and effects. Thus, we contend that agent-based models belong to a broader class of system-based models, which are models specifically intended to represent systems composed of interacting elements. In the demographic context, any human population is in fact composed of multiple interacting levels of complexity: individuals, social groups, institutions, etc., any and all of which are suitably complex for in-depth investigation in their own right.

By acknowledging this fundamental aspect of the social realm, demography can continue to shift toward modelbased science by making those interactions between different elements of the population an explicit object of scientific interest. This then instantiates system-based models in demography as a method for representing interacting, complex behaviours and investigating how these interactions shape demographic change. Re-casting demographic model-building in this way has clear ramifications for future efforts to build stronger theories regarding population change.

This is not to say that system-based approaches are the only option - and indeed, numerous demographic problems will still be addressed perfectly adequately with previous, well-established modelling paradigms. Further, systembased models are somewhat dependent on the presence of sensible theories regarding social systems, and such theories are very difficult to formalise (Kluver et al., 2003; Moss and Edmonds, 2005). A possible way out of this difficulty may be to reconnect system-based approaches to a classical scientific research programme which promotes a functionalmechanistic analysis of populations (Franck, 2002a).

\section{System-Based Modelling}

Bringing all of these elements together, we propose that demography is in need of a fifth paradigm, one which maximises the strengths of empirical demographic research as well as the flexibility of simulation methods. While agentbased methods have limitations of their own, as outlined above, this new paradigm integrating these methods into the empirical and inductive research programme of demography may allow us to surpass these difficulties. To do so, we must shift toward a new scientific object in demography: interactions.

Therefore we have proposed a fifth, new paradigm for demography: system-based modelling. This approach is based on a functional-mechanistic research programme, which we discuss in more detail in the next section. Agent-based 
modelling is of course a possible methodology for implementing a system-based modelling approach, but the overall paradigm need not be tied solely to one methodology. In essence, we propose that demography should seek to examine the interactions between elements of a given population, and the mechanisms driving them. These elements can be described via formal models derived from observation using inductive methods. We posit that this paradigm forms a natural extension of the previous four, and also broadens the scope of demographic research. Following Franck (2002a) and Burch (2003b), we also posit that the system-based approach can enhance the theoretical base of demography by allowing for the development of formal conceptual models.

The strength of simulation-based scenario generation approaches provides one illustration of the potential for this kind of methodological innovation. Demography, as we have seen, has a particular strength in its ability to connect directly to practical, policy-relevant issues (Xie, 2000; Morgan and Lynch, 2001). Methodological enhancements like those outlined above can push this particular strength even further. Bayesian approaches allow for formal statistical decision analysis, and thus support efforts in planning (Bijak, 2010). System-based approaches, when coupled closely with statistical analysis techniques, provides a simulated environment in which policy-makers can investigate a range of possible policy changes. This kind of experimentation is made possible by coherent scenario generation, in which the behaviour of simulated individuals closely follows empirical patterns for statistical individuals observed via demographic data (Courgeau, 2012).

The predictive horizon for demography is thought to be approximately one generation (Keyfitz, 1981), so scenario generation augmented with statistical analysis of model results allows for the exploration of more possible futures (Bijak, 2010). Utilising well-constructed simulation models to investigate these scenarios allows us to examine interactions that drive macro-level patterns of population change in more detail, while also removing some of the limitations of traditional data-heavy statistical methodologies (Silverman et al., 2011). Thus, this application of a system-based approach demonstrates the benefits of an effective combination of the empirical strengths of demography with simulation: demographers can now study complex interactions and behaviours in artificial populations which allow for coherent scenario generation. We have already seen some examples of this type of inductive, empirical system-based approach in the literature - see Klabunde (2014).

\section{A Functional-Mechanistic Research Programme for Demography}

Having established the need for, and utility of, a systembased approach in demography, how can we ensure that this new paradigm conforms to a productive classical scientific research programme? We see such an approach extant in

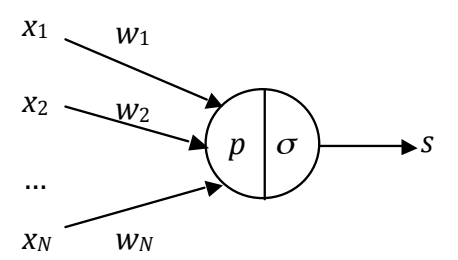

Figure 1: The McCulloch and Pitts (1943) model of a single neuron. Adapted from Franck (2002: 143).

the natural sciences since Francis Bacon. We propose that a move toward system-based modelling as a fifth paradigm presents an opportunity to reconnect with the classical scientific research programme, and in so doing, establish demography as a true model-based science rather than a 'science in the making' (Courgeau and Franck, 2007).

As an illustrative example, consider the famous neural model produced by McCulloch and Pitts (1943), pictured in Figure 1. This model was enormously influential, and led to the development of artificial neural networks some years later. When we look closely at this model, what is actually represented by this simplified neuron?

This model does not represent the physical structure or chemical behaviour of the neuron - we see no representation of the neuron's shape, its genetic material, membrane, etc. What McCulloch and Pitts did was to observe the behaviour of the neuron and represent its functional architecture, without which we would not see its main properties come about. They identified five main functions, which can be seen in Figure 1: receiving input stimuli; weighting inputs with synaptic coefficients; calculating the sum of weighted inputs $(p)$; fixing a threshold of stimulation below which no signal is transmitted; and calculating the exit signal $s$.

Thus, this neuron model represents the functional structure of the processes that generate the observed behaviour of a neuron. However, the model ignores the causal factors underlying those individual functions - the model is entirely a formal, conceptual construction.

In one sense, McCulloch and Pitts have followed a process similar to reverse engineering: they have induced the design of the neuron from its end products. They of course have no interest in reproducing the neuron physically, and yet they followed much the same process as engineers seeking to reconstruct the design of a device from the final products. In doing so, they have inferred the structure of the essential functions of a neuron based on its behaviour (Franck, 2002b).

This method is essentially derived from the classical programme of scientific research, in which scientists try to in- 
fer (induce) the functional structure which rules the process generating the property of interest ${ }^{1}$. We refer to this method here as a functional-mechanistic one, as it attempts to model the structure of functions that rule a specific mechanism.

When examining social properties, we must similarly model the structure of social functions that drive social processes which generate those properties, and which are necessary to generate those properties. For example, work in demography has established that some combination of fertility, mortality and migration generate variations in population structure. Similarly, the law of supply and demand was inferred from the observation of markets and the social exchanges which generate them. Karl Marx's study of industrial production and its social organisation led him to infer the general structure of functions which drive this process - and in doing so develop the principle of separating labour and capital. These well-known results of social science were all derived from the application of this classical, functional-mechanistic programme of science.

Once we have established the functional structure underlying the process which generates our social property, we may use this as a means to identify and model social factors that have contributed this process. In doing so we may restrict ourselves to the investigation of factors which have contributed directly to the combination of functions we have uncovered. As an example, demographers take mortality, fertility and migration as the functions which define its scientific object - population change - and thus they undertake their investigations on the empirical study of these processes and social factors related to them.

We thus propose that the new system-based paradigm for demography should follow this functional-mechanistic approach. Model-building should follow a process of collecting all relevant empirical information about the social property of interest, which then allows us to uncover the social functions required by this property. Once this functional structure has been modelled, we can then proceed to the modelling of the factors which have contributed to the process which generated this social process, individual agents, institutions, groups, and so on (Franck, 2002a).

In the case of traditional demographic examinations of the key processes of mortality, fertility and migration, we can see clearly that the four previous paradigms are sufficient to answer a great deal of questions related to these processes. They also allow us to identify the presence of important interactions between various elements of human populations that contribute to these processes. Once we turn our investigations directly toward these interactions, rather than the results of these interactions as part of those key processes, then we are shifting our scientific object - and thus we need to follow the system-based approach. Simulation methods

\footnotetext{
${ }^{1}$ The type of induction we refer to here is drawn from Francis Bacon, in which induction proceeds similarly to reverse engineering as described above. See Franck (2002a) for further explication.
}

can allow us to represent these interactions explicitly in a social context defined by our best available knowledge regarding the induced functional-mechanistic structure of the social system, and then investigate the potential impact of changes in these interactions.

\section{The Process of System-Based Modelling}

The system-based modelling paradigm proposed here brings with it significant advantages: the paradigm expands upon the previous four while incorporating insights from modelbased science in other disciplines. Bringing this paradigm forward will require some substantial demands upon the demographic community, however; system-based modelling requires not only new conceptual developments regarding the relationships and interactions between elements of population systems, but also requires the development and use of new tools which are complex and often difficult to build and to analyse.

Thus we propose that the system-based paradigm would take shape as a four-step process, following on from Franck (General Conclusion 2002a):

1. Observation of the properties of a given population (data)

2. Inference of the formal structure implied by these properties

3. Using (2) as a guide to investigating social mechanisms that generate properties

4. Model-based investigation of formal structures to seek verification with observed data

Note that statistical modelling and uncertainty qualification could serve as a means to infer the formal structures from data in steps (1) and (2).

As we have seen, multilevel modelling in demography has already provided a means to develop a greater insight into certain aspects of complex population systems by demonstrating the existence of interactions between parts of those systems. System-based modelling allows us to move forward once more by allowing us to explore these interactions as the central object of study. Simulations allow us to explore the the social factors that interest us, and generate scenarios which increase our understanding of those factors. These scenarios could represent everything from individual alterations in behaviour at the agent level, to changes in policy at a societal level (as in Silverman et al. (2013)). Using simulations in such a way also allows us to examine the non-linear impact of changes to complex, interacting social systems - a key element in the behaviour of social systems which is not possible to represent using conventional mathematical models.

In practice, system-based population modelling can rely to some extent on the existing agent-based approaches, insofar as they are subjected to the inductive principles of the 
scientific method. However, a key open question remains: what principles should be followed to illuminate the inductive construction of such models? We suggest that this question is fundamental to the future direction of demography, and we have no doubt that vigorous and open debate on this question will be a vital part of efforts to take demography in a new direction.

\section{Lessons for Social Simulation}

System-based modelling will present some significant challenges for demographers in order to take advantage of these potential benefits. However, social simulation researchers are by no means exempt from this requirement to adjust their methods in response to the changing landscape in demography. For those of us who wish to use social simulation to investigate real-world populations and their properties, we may expect that the recent developments in demography will have significant impact on our research methodologies.

Looking at the field as a whole, social simulation is a continually growing area of research which continues to establish new links with numerous and varied areas of social science. The attraction of simulation approaches, particularly agent-based models, for social scientists is undeniable; the prospect of simulating individual behaviour and being able to observe the resulting macro-level effects is enticing to not only demographers, but political scientists and economists, among others.

What we have proposed here is admittedly very ambitious - our proposed fifth paradigm has implications for all of social science, in certain respects, and demands a new level of engagement between social simulation practitioners with social scientists. We argue here that the payoff for such engagement will be significant, resulting in stronger theoretical frameworks for social scientists and more empirical relevance for social simulation.

Examining the history of demography has revealed however that such a change would not obviate the need for social simulation as a separate discipline. In demography, the previous four paradigms of research remain very much present, and indeed our proposed fifth paradigm would not be suitable for certain demographic questions that are still best answered using other methods. Likewise, system-based methods may be used as vehicles for broader philosophical investigation of social systems, or as a type of opaque thought experiment (Di Paolo et al., 2000), or for many other purposes beyond prediction or the direct study of population change (Epstein, 2008). Therefore we can expect social simulation to remain and to flourish as a discipline making unique contributions to big questions about sociality in general.

However, successful application of the system-based paradigm in demography will require perhaps more methodological shifts from the social simulation side than is commonly accepted. The application of inductive, empirical methods to the simulation of human populations remains a significant challenge, and requires a different approach more firmly connected to empirical investigation and traditional scientific approaches. We hope that our elucidation of this approach and its potential benefits and pitfalls may help drive these efforts forward, and eventually lead to a productive system-based approach that gives us a greater understanding of population change.

\section{Acknowledgements}

Eric Silverman and Jakub Bijak gratefully acknowledge the Engineering and Physical Sciences Research Council (EPSRC) grant EP/H021698/1 Care Life Cycle, funded within the Complexity Science in the Real World theme.

\section{References}

Aalen, O. (1975). Statistical inference for a family of counting processes. $\mathrm{PhD}$ thesis, Berkeley: University of California.

Agazzi, E. (1985). Commensurability, incommensurability, and cumulativity in scientific knowledge,. Erkenntniss, , 22:5177.

Bijak, J. (2010). Forecasting International Migration in Europe: A Bayesian View. Springer Series on Demographic Methods and Population Analysis, Vol. 24. Dordrecht: Springer.

Bijak, J., Hilton, J., Silverman, E., and Cao, V. (2013). Reforging the wedding ring: Exploring a semi-artificial model of population for the united kingdom with gaussian process emulators. Demographic Research, 29(27):729-766.

Billari, F. and Prskawetz, A. (2003). Agent-Based Computational Demography: Using simulation to improve our understanding of demographic behaviour. New York: Physica Verlag.

Bullock, S. and Silverman, E. (2008). Levins and the legitimacy of artificial worlds. Epistemological Perspectives on Simulation, Lisbon, Portugal.

Burch, T. (2003a). Data, models, theory and reality: the structure of demographic knowledge. In Billari, F. and Prskawetz, A., editors, Agent-Based Computational Demography: Using simulation to improve our understanding of demographic behaviour, pages 19-40. Physica-Verlag, New York.

Burch, T. (2003b). Demography in a new key: A theory of population theory. Demographic Research, 9(11):263-284.

Chattoe, F. (2003). The role of agent-based models in demographic explanation. In Billari, F. and Prskawetz, A., editors, AgentBased Computational Demography: Using simulation to improve our understanding of demographic behaviour, pages 41-54. Physica-Verlag, New York.

Clark, S., Thomas, J., and Bao, L. (2012). Estimates of age-specific reductions in hiv prevalence in uganda: Bayesian melding estimation and probabilistic population forecast with an hivenabled cohort component projection model. Demographic Research, 27(26):743-774.

Courgeau, D. (2007). Multilevel synthesis: From the group to the individual. Dordrecht: Springer. 
Courgeau, D. (2010). Paradigmes démographiques et cumulativité. In Walliser, B., editor, La cumulativité du savoir en sciences sociales, pages 243-276. Editions de l'EHESS, Paris.

Courgeau, D. (2012). Probability and Social Science: Methodological relationships between the two approaches. Methodos Series 10 , Springer.

Courgeau, D. and Franck, R. (2007). Demography: A fully-formed science or a science in the making? Population-E, 62(1):3946.

Di Paolo, E., Noble, J., and Bullock, S. (2000). Simulation models as opaque thought experiments. In Bedau, M., McCaskill, J., Packard, N., and Rasmussen, S., editors, Proceedings of the Seventh International Conference on Artificial Life, pages 497-506. MIT Press, Cambridge, MA.

Epstein, J. (2008). Why model? Journal of Artificial Societies and Social Simulation, 11(4):12.

Franck, R., E. (2002a). The Explanatory Power of Models: Bridging the gap between empirical and theoretical research in the social sciences. Kluwer Academic Publishers.

Franck, R. (2002b). Computer simulation and the reverse engineering method: Conclusions of part ii. In Franck, R., editor, The Explanatory Power of Models. Methodos Series vol 1, pages 141-146. Kluwer Academic Publishers, Dordrecht/Boston/London.

Gneiting, T. and Raftery, A. (2007). Strictly proper scoring rules, prediction, and estimation. Journal of the American Statistical Association, 102(477):359-378.

Godfrey-Smith, P. (2006). The strategy of model-based science. Biology and Philosophy, 21(5):725-740.

Granger, G.-G. (1994). Formes, opéruptions, objets. Librairie Philosophique Vrin.

Graunt, J. (1662). Natural and political observations mentioned in a following index, and made upon the bills of mortality. The Roycroft, London.

Henry, L. (1959). D'un probeème fondamental de l'analyse démographique. Population, 14(1):9-32.

Kennedy, M. and O'Hagan, T. (2001). Bayesian calibration of computer models. Journal of the Royal Statistical Society, Series $B, 63(3): 425-464$.

Keyfitz, N. (1981). The limits of population forecasting. Population and Development Review, 7(4):579-593.

Klabunde, A. (2014). Computational economic modelling of migration. Ruhr Economic Papers, 471.

Kluver, J., Stoica, C., and Schmidt, J. (2003). Formal models, social theory and computer simulations: Some methodological reflections. Journal of Artificial Societies and Social Simulation, 6(2):http://jasss.soc.surrey.ac.uk/6/2/8.html.

Kuhn, T. (1962). The Structure of Scientific Revolutions. University of Chicago Press.

Kuhn, T. (1970). Postscript, The Structure of Scientific Revolutions. University of Chicago Press.
Luhmann, N. (1984). Social Systems. Stanford University Press.

Lutz, W. (2013). Demographic metabolism: A predictive theory of socio-economic change. Population and Development Review, 38(Supplement):283-301.

McCulloch, W. and Pitts, W. (1943). A logical calculus of the ideas immanent in nervous activity. Bulletin of Mathematical Biophysics, 5(4):115-133.

Morgan, S. and Lynch, S. (2001). Success and future of demography: The role of data and methods. Annals of the New York Academy of Sciences, 954:35-51.

Moss, S. and Edmonds, B. (2005). Towards good social science. Journal of Artificial Societies and Social Simulation, 8(4):http://jasss.soc.surrey.ac.uk/8/4/13.html.

O'Hagan, A. (2006). Bayesian analysis of computer code outputs: a tutorial. Reliability Engineering and System Safety, 91(1011):1290-1300

Poole, D. and Raftery, A. (2000). Inference for deterministic simulation models: The bayesian melding approach. Journal of the American Statistical Association, 95(452):1244-1255.

Raftery, A. (1995). Bayesian model selection in social research. Sociological Methodology, 25:111-163.

Raftery, A., Li, N., Seveikova, H., Gerland, P., and Heilig, G (2012). Bayesian probabilistic population projections for all countries. Proceedings of the National Academy of Sciences, 109:13915-13921.

Ryder, N. (1951). The cohort approach: Essays in the measurement of temporal variations in demographic behaviour. $\mathrm{PhD}$ thesis, New York: Princeton University.

Schelling, T. (1978). Micromotives and macrobehavior. W.W. Norton.

Silverman, E., Bijak, J., and Noble, J. (2011). Feeding the beast: Can computational demographic models free us from the tyranny of data? In Lenaerts, T., Giacobini, M., Bersini, H., Bourgine, P., Dorigo, M., and Doursat, R., editors, Advances in Artificial Life, ECAL 2011, pages 747-754. MIT Press, Cambridge, MA.

Silverman, E. and Bryden, J. (2007). From artificial societies to new social science theory. In e Costa, F. A., Rocha, L., Costa, E., Harvey, I., and Coutinho, A., editors, Advances in Artificial Life, 9th European Conference, ECAL 2007 Proceedings, pages 645-654. Springer, Berlin-Heidelberg.

Silverman, E., Hilton, J., Noble, J., and Bijak, J. (2013). Simulating the cost of social care in an ageing population. In Rekdalsbakken, W., Bye, R., and Zhang, H., editors, Proceedings of the 27th European Conference on Modelling and Simulation, pages 689-695. Digitaldruck Pirrot, Dudweiler.

Xie, Y. (2000). Demography: Past, present and future. Journal of the American Statistical Association, 95(450):670-673. 